\title{
REJEITO DA MINERAÇÃO DE AREIA COMO MATERIAL CIMENTÍCIO SUPLEMENTAR
}

\author{
FIGUEIREDO, ALINE SANTANA \\ Mestranda em Engenharia Civil \\ Universidade Federal de Ouro Preto \\ Minas Gerais; Brasil \\ alinesantanafigueiredo@gmail.com
}

\author{
KUSTER, LUANA DRAGO \\ Graduanda em Engenharia Civil \\ Universidade Federal de Ouro Preto \\ Minas Gerais; Brasil \\ luanadkuster@gmail.com
}

\author{
FONTES, WANNA CARVALHO \\ Arquiteta e Urbanista, D.Sc \\ Universidade Federal de Ouro Preto \\ Minas Gerais; Brasil \\ wanna.fontes@ufop.edu.br
}

\author{
SANTOS, BRUNA LOURES DE CASTRO \\ Graduanda em Arquiterura e Urbanismo \\ Universidade Federal de Ouro Preto \\ Minas Gerais; Brasil \\ brunalouresc@gmail.com
}

\author{
ANTUNES, LUDIMILA GOMES \\ Graduanda em Arquiterura e Urbanismo \\ Universidade Federal de Ouro Preto \\ Minas Gerais; Brasil \\ ludimila.antunes@aluno.ufop.edu.br
}

\author{
PEIXOTO, RICARDO ANDRÉ FIOROTTI \\ Engenheiro Civil, D.Sc \\ Universidade Federal de Ouro Preto \\ Minas Gerais; Brasil \\ fiorotti.ricardo@gmail.com
}

\section{RESUMO}

$\mathrm{O}$ rejeito proveniente do processamento de material granular destinado à produção de areia industrial e para construção civil é gerado em grande quantidade no Brasil e ainda inexplorado cientificamente. Denominado goma de areia (GA), é um material altamente fino e que representa ônus ao processo de produção. Esse estudo avaliou as características físicas, químicas, mineralógicas e morfológicas da goma de areia, assim como sua aplicação em concretos; com o objetivo de promover sua reinserção na cadeia produtiva. Realizadas as análises propostas, verificou-se na literatura que esse resíduo possui características desejáveis para aplicação como material cimentício suplementar (SCM), incluindo suas propriedades físicas e químicas; como distribuição granulométrica, teores de óxidos presentes, composição de fases e teor de amorfos, visto que todos estes fatores afetam a interação do material com o cimento. Através da avaliação dos concretos produzidos, notou-se que a goma de areia proporcionou melhoria das propriedades físicas e mecânicas das matrizes, apresentando potencial aumento na durabilidade e na resistência a meios agressivos, prevenindo o surgimento de patologias.

Palavras-chave: material cimentício suplementar, tecnologia do concreto, rejeito, sustentabilidade.

\section{ABSTRACT}

The waste from the processing of granular material for the production of industrial sand and aggregates for civil construction is generated in large quantities in Brazil and still untapped scientifically. Sand mining tailings (ST) is a highly fine particulate material that represents onus to the production process. This study evaluated the physical, chemical, mineralogical and morphological characteristics of ST, as well as its application in concrete; in order to promote their reinsertion in the production chain. After the proposed analyzes, it was found in the literature that this residue has desirable characteristics for application as supplementary cementitious material (SCM), including their physical and chemical properties such as particle size distribution, oxide contents, phase composition and amorphous content, all of which affect the interaction between the material and Portland cement. The evaluation of produced concretes showed that ST improved the physical and mechanical properties of the matrices, presenting a potential increase in durability and resistance to aggressive environments, preventing the appearance of pathologies.

Keywords: supplementary cementitious material, concrete technology, tailings, sustainability.

\section{INTRODUÇÃO}

A atividade mineradora é responsável por um expressivo impacto ambiental e social, desde a extração de recursos naturais não renováveis até a disposição final de seus resíduos; que pode implicar no desmatamento de grandes extensões territoriais, processos erosivos e na contaminação do solo e de cursos d'água (YOUNG e YANG, 2019) 
(LOTTERMOSER, 2006). Desta forma, mesmo após a interrupção das atividades de mineração, pode haver a contaminação do solo e das águas superficiais ou subterrâneas por metais pesados e outras substâncias provenientes da atividade de extração, beneficiamento e refino mineral, seja por dispersão na atmosfera ou por lixiviação (FIGUEIREDO, CAPITANI e GITAHY, 2004) (MECHI e SANCHES, 2010).

Dentre as atividades mineradoras brasileiras, a extração da areia foi uma atividade que se intensificou no país ao longo dos anos, juntamente com setor da construção civil. De acordo com a Associação Nacional das Entidades de Produtores de Agregados para Construção (ANEPAC, 2019), a extração de areia teve um aumento de 118,4\% entre os anos de 2000 e 2014, chegando a 349 milhões de toneladas produzidas neste ano, representando cerca de 1,8 toneladas per capita (DNPM, 2016). A areia é utilizada na construção civil como agregado miúdo para concretos, argamassas, base de pavimentos rígidos e flexíveis, dentre outros, sendo definida na indústria como um bem mineral granular, com tamanho em um intervalo definido (de 2 a 0,06 mm) (ANEPAC, 2019) (CHAVES e WHITAKER, 2012). Trata-se de um material que possui composição silicática, com predominância de quartzo (DNPM, 2016), podendo ser obtida a partir de depósitos em leitos de rios e planícies aluviais, rochas sedimentares e mantos de alteração de rochas cristalinas (ANEPAC, 2019).

De acordo com o tipo de depósito mineral, é definido o processo de lavra; que pode ser através de desmonte hidráulico, escarificação ou simplesmente por dragagem (ANEPAC, 2019) (CHAVES e WHITAKER, 2012). No processo de dragagem a extração é feita no interior de uma cava preenchida com água, através de uma draga instalada em uma estrutura externa. Esse equipamento apresenta bombas que conduzem a polpa formada por água e areia até os silos e às instalações de lavagem (ANEPAC, 2019). Após esse procedimento, ocorre a separação entre a areia e o rejeito, finos constituídos basicamente por silte e argila, feita pela diferença de densidade entre os produtos (ANEPAC, 2019) (WHITAKER, BRAGA e CHAVES, 2002). O rejeito resultante do processo, denominado goma de areia (GA), pode representar até $20 \%$ da produção da jazida (FERREIRA e DAITX, 2003). É um resíduo indesejável por apresentar uma granulometria imprópria para aplicação como agregado em argamassas ou concretos e por ser, também, fonte de contaminação de alumínio, ferro e álcalis, na fabricação de vidros especiais e de produtos químicos (LUZ e LINS, 2005). Uma vez que este ainda não possui valor agregado na indústria, representa um dos principais problemas ambientais na mineração de areia industrial (FERREIRA, 1997) (BITAR, IYOMASA e JR., 2000).

A incorporação da GA em matrizes cimentícias como material cimentício suplementar (SCM) contribui para a reinserção desse rejeito na cadeia produtiva, redução no consumo de ligante (cimento Portland) e a consequente redução da emissão de $\mathrm{CO} 2$ relacionada à sua produção, diminuição do consumo de recursos naturais não renováveis e redução da criação de reservatórios destinados ao armazenamento desse rejeito (YANG, JUNG, et al., 2015) (JUENGER e SIDDIQUE, 2015). Muitos pesquisadores tem estudado sobre a aplicação de resíduos industriais e da mineração incorporados às matrizes cimentícias, com resultados satisfatórios referentes ao desempenho mecânico e durabilidade desses compósitos (JUENGER e SIDDIQUE, 2015) (ANDRADE, 2018) (JUENGER, SNELLINGS e BERNAL, 2019) (COSTA, 2019). Rahla et al. (2019) propõem a avaliação do desempenho ambiental, econômico e funcional de concretos com adição de cinzas volantes, escória de alto forno e sílica ativa em diferentes percentuais de substituição ao cimento Portland a partir de uma lista de quinze indicadores de sustentabilidade, foram obtidos resultados que indicam uma boa ecoeficiência dessas matrizes. Similarmente, Carvalho et al. (2019) analisou a incorporação de resíduos industriais e da mineração para a produção de cimentos com emissão reduzida de $\mathrm{CO} 2$, obtendo um cimento belítico inteiramente composto por resíduos e com temperatura de calcinação do clínquer $200^{\circ} \mathrm{C}$ mais baixa do que um cimento convencional.

Snellings (2016) enfatiza a urgência de se intensificar os vínculos entre a gestão de resíduos e a produção de materiais de construção, visto que existe uma demanda crescente por uma economia mais circular e com emissão reduzida de carbono. Aprianti (2017) apresenta uma revisão a respeito do uso bem-sucedido de resíduos industriais e da agricultura, como cinzas volantes, escória, sílica ativa, cinza de casca de arroz, cinza de óleo de palma, cinza de bagaço de cana de açúcar, cinza de madeira, cinza de bambu e cinzas de sabugo de milho na indústria de concreto; indicando que o aprimoramento do conhecimento existente e a investigação de outros resíduos a serem utilizados como SCM serão uma contribuição valiosa e uma solução viável na construção sustentável. Desta forma, esse estudo busca o melhor entendimento do rejeito GA através de caracterizações física, química e mineralógica desse material. Esse estudo visa contribuir com a criação de um banco de dados de caracterização e composição desse rejeito, possibilitando o desenvolvimento de novos estudos e possíveis aplicações, visto que esse material ainda se encontra pouco conhecido cientificamente. 


\section{MATERIAIS E MÉTODOS}

Nesta pesquisa, utilizou-se goma de areia proveniente do Rio Grande do Sul, Brasil. O material foi obtido na forma de lama, como se observa na Figura 1a. Após o recebimento do material em seu estado natural, a GA foi seca em estufa a uma temperatura de $100^{\circ} \mathrm{C} \pm 5^{\circ} \mathrm{C}$. Esse procedimento ocorreu durante 2 dias e foi interrompido no momento em que o material atingiu constância de massa. Com o objetivo de desaglomerar os grãos do rejeito (SANTOS, 1975), a GA foi submetida a um procedimento manual de destorroamento em almofariz de porcelana, conforme figura $1 \mathrm{~b}$.

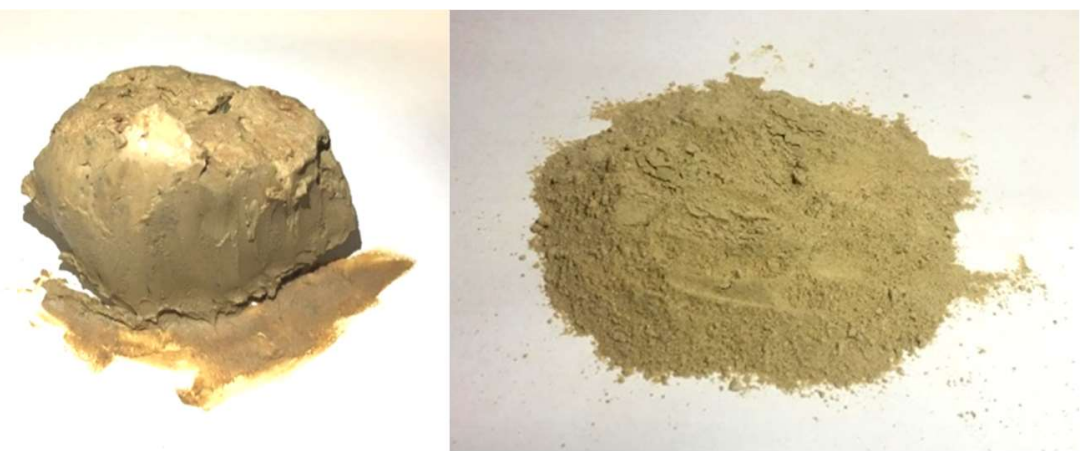

Figura 1: (a) Goma de areia em seu estado natural (b) Goma de areia após o beneficiamento

\subsection{Caracterização física, química e mineralógica do rejeito}

Amostras de GA foram submetidas ao ensaio de granulometria por difração de laser em equipamento BETTERSIZER 2000. Essa técnica possibilitou a obtenção das dimensões características D10, D50 e D90 e a distribuição granulométrica do rejeito. A massa unitária foi definida através dos procedimentos indicados pela NBR NM 45 (ABNT, 2006) e a massa específica da GA foi determinada de acordo com as prescrições da NBR 16605 (ABNT, 2017) para cimento Portland e outros materiais em pó, por meio do frasco volumétrico de Le Chatelier.

A avaliação química do rejeito foi realizada utilizando a técnica de Fluorescência de Raios-X (FRX) em equipamento Panalytical Epsilon 3x, com a finalidade de determinar os teores de óxidos presentes nesse material e contribuir para a análise e interpretação dos dados obtidos na técnica de Difração de Raios-X (DRX).

A avaliação mineralógica foi procedida por ensaio de Difração de Raios-X (DRX) em equipamento Bruker D2 Phaser, com radiação $\mathrm{CuK} \alpha$, faixa de varredura $2 \theta$ de $6-70^{\circ}$, passo de $0,018^{\circ}$ a cada segundo, com o propósito de verificar as diferentes estruturas cristalográficas nas quais se dispõem os elementos verificados na Fluorescência de Raios-X. Para a identificação das principais fases mineralógicas, foram utilizados o software PANalytical X'pert HighScore Plus V3.0. A análise quantitativa foi realizada através do método de Rietveld no mesmo software, utilizando o banco de dados ICSD (Inorganic Crystal Structure Database) (LEIBNIZ ASSOCIATION, 2019). Para este fim, adicionou-se 20\%, em massa, de fluoreto de Lítio (griceita) à amostra, objetivando identificar o teor de material amorfo presente em sua composição.

Com o objetivo de identificar a composição do rejeito através da perda de massa associada a picos exotérmicos ou endotérmicos foi executada a análise Termogravimétrica e Termodiferencial (TG/DTA). Essa técnica foi realizada em equipamento Shimadzu DTG- $60 \mathrm{H}$, com aquecimento de $25^{\circ} \mathrm{C}$ a $1000^{\circ} \mathrm{C}$, a uma taxa de $10^{\circ} \mathrm{C} / \mathrm{min}$, discretizados a $5^{\circ} \mathrm{C} / \mathrm{min}$ os intervalos entre $400^{\circ} \mathrm{C}$ e $600^{\circ} \mathrm{C}$ e entre $900^{\circ} \mathrm{C}$ e $1000^{\circ} \mathrm{C}$; em atmosfera inerte de $\mathrm{N} 2(\mu 1 / \mathrm{min})$.

\subsection{Aplicação em concretos}

Afim de se verificar a viabilidade técnica de aplicação da GA em matrizes cimentícias, foram moldados corpos de prova cilíndricos (50x100mm) de concreto, de acordo com o preconizado pela NBR 5738 (ABNT, 2015), com (GA) e sem adição (RF) do material proposto. Foi utilizado aditivo superplastificante à base de policarboxilato (PowerFlow 4001, MC Bauchemie) para manutenção do fator água/cimento e fixação de determinados parâmetros, com o intuito de minimizar as variáveis experimentais; sendo estes o consumo de cimento por metro cúbico de concreto e a trabalhabilidade da mistura no estrado fresco, determinada através do ensaio de abatimento do tronco de cone conforme normatizado pela NBR NM 67 (ABNT, 1998), para ambas as dosagens. O concreto GA possui 16\% de adição de goma de areia em relação à massa de cimento e a proporção dos materiais foi feita conforme apresentado na Tabela 1 , a seguir. 
Tabela 1 - Proporcionamento dos materiais para cada dosagem

\begin{tabular}{c|c|c|c|c|c|c|c}
\hline Dosagem & $\begin{array}{c}\mathbf{C P V} \\
\left(\mathbf{k g} / \mathbf{m}^{3}\right)\end{array}$ & $\begin{array}{c}\mathbf{G A} \\
\left(\mathbf{k g} / \mathbf{m}^{3}\right)\end{array}$ & $\begin{array}{c}\text { Agregado } \\
\text { miúdo } \\
\left(\mathbf{k g} / \mathbf{m}^{3}\right)\end{array}$ & $\begin{array}{c}\text { Agregado } \\
\text { graúdo } \\
\left(\mathbf{k g} / \mathbf{m}^{3}\right)\end{array}$ & $\begin{array}{c}\text { Água } \\
\left(\mathbf{k g} / \mathbf{m}^{3}\right)\end{array}$ & $\begin{array}{c}\text { Superplastificante } \\
\mathbf{( \% )}\end{array}$ & $\begin{array}{c}\text { Abatimento } \\
\text { do tronco de } \\
\text { cone } \mathbf{( c m )} \\
(\mathbf{N B R} \text { NM 67) }\end{array}$ \\
\hline RF & 350 & - & 865 & 955 & 193 & 0,4 & 9,0 \\
\hline GA & 350 & 55 & 834 & 930 & 193 & 1,7 & 9,0 \\
\hline
\end{tabular}

A cura dos corpos de prova foi realizada em câmara úmida durante 28 dias, sob temperatura de $25 \pm 2^{\circ} \mathrm{C}$ e umidade relativa de $90 \% \pm 5 \%$. No estado endurecido, foi procedida a caracterização física através do ensaio de massa específica, índice de vazios e absorção de água, de acordo com a NBR 9778 (ABNT, 2005) e determinação da velocidade de pulso ultrassônico (VPU), conforme preconizado pela NBR 8802 (ABNT, 2019). A caracterização mecânica foi realizada por meio de ensaios de resistência à compressão e de resistência à tração por compressão diametral, segundo NBR 5739 (ABNT, 2018) e NBR 7222 (ABNT, 2011), respectivamente.

\section{RESULTADOS E DISCUSSÃO}

\subsection{Propriedades físicas}

Após o procedimento manual de destorroamento, as dimensões características D90, D50 e D10 foram obtidas por meio da granulometria por difração de laser (Tabela 2).

Tabela 2 - Propriedades físicas da goma de areia

\begin{tabular}{c|c|c|c|c}
\hline $\begin{array}{c}\mathrm{D} 90 \\
(\mu \mathrm{m})\end{array}$ & $\begin{array}{c}\mathrm{D} 50 \\
(\mu \mathrm{m})\end{array}$ & $\begin{array}{c}\mathrm{D} 10 \\
(\mu \mathrm{m})\end{array}$ & $\begin{array}{c}\text { Massa específica } \\
\left(\mathrm{g} / \mathrm{cm}^{3}\right)\end{array}$ & $\begin{array}{c}\text { Massa unitária } \\
\left(\mathrm{g} / \mathrm{cm}^{3}\right)\end{array}$ \\
\hline 25,0 & 6,5 & 1,3 & 2,6 & 0,8 \\
\hline
\end{tabular}

De acordo com os resultados obtidos, verifica-se que a GA é um material pulverulento altamente fino; com $90 \%$ das partículas abaixo de $20,0 \mu \mathrm{m}$ e $50 \%$ abaixo de $6,5 \mu \mathrm{m}$. Devido à gama diversificada de SCMs utilizada, as relações genéricas entre composição, tamanho de partícula, condições de exposição como temperatura ou umidade relativa tornamse cada vez mais cruciais (LOTHENBACH, SCRIVENER e HOOTON, 2011) (ARVANITI, JUENGER, et al., 2015).

A ASTM C618 (ASTM, 2019), que especifica limites para cinzas volantes e pozolanas naturais ou calcinadas em concretos, permite a retenção de até $34 \%$ do material na peneira $\mathrm{n}^{\circ} 325(45 \mu \mathrm{m})$; quantidade maior do que a estabelecida pela NBR 5752 (ABNT, 2014), que determina o desempenho de materiais pozolânicos com cimento Portland aos 28 dias (até 20\%) e pela NBR 15894-1 (ABNT, 2010), que define requisitos para incorporação do metacaulim ao cimento Portland (até $10 \%$ ). A GA está de acordo com todos os limites estabelecidos.

De acordo com as normas mencionadas e partindo do ponto de vista de que a finura influencia diretamente na reatividade dos SCMs, além de sua consequente interação com determinados compostos do cimento Portland (KORPA, KOWALD e TRETTIN, 2008) (SNELLINGS, MERTENS e ELSEN, 2012), esse rejeito pode então se apresentar como um eficiente SCM.

\subsection{Propriedades morfológicas}

A imagem obtida através da microscopia eletrônica de varredura (MEV) complementa os resultados da granulometria por difração de laser. As partículas de GA possuem formato irregular, mas com uma certa uniformidade na proporção de suas dimensões. Há partículas de tamanhos variados, entre aproximadamente 2,0 e 25,0 $\mu \mathrm{m}$, e a presença de aglomerações de partículas finas (Figura 2). 


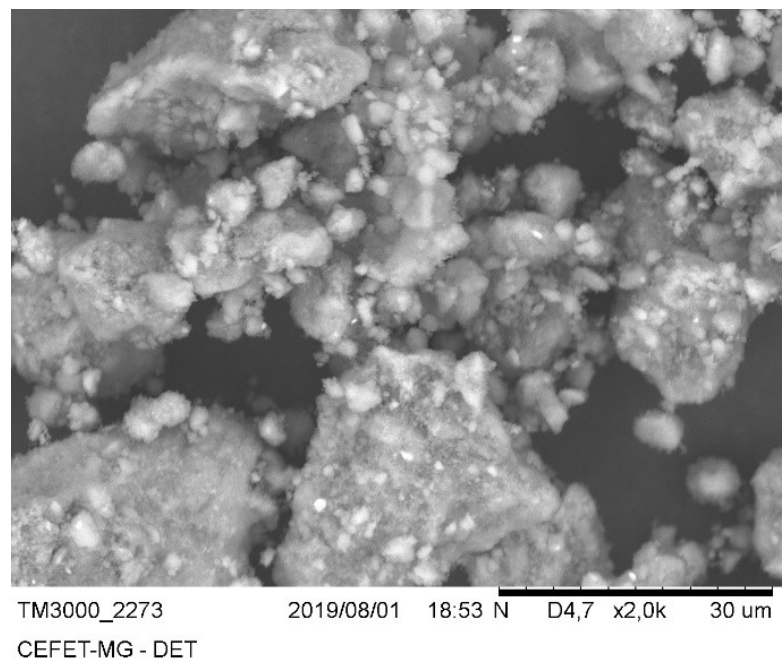

Figura 2 - Imagem da goma de areia obtida através de MEV

\subsection{Propriedades químicas e mineralógicas}

Os principais constituintes químicos da GA observados na análise de FRX (em óxidos) foram silício $\left(\mathrm{SiO}_{2}\right)$, a alumínio $\left(\mathrm{Al}_{2} \mathrm{O}_{3}\right)$ e ferro $\left(\mathrm{Fe}_{2} \mathrm{O}_{3}\right)$ (Tabela 3). Segundo a ASTM C618 (ASTM, 2019), que define as diretrizes para materiais pozolânicos incorporados em matrizes cimentícias, a soma dos teores de dióxido de silício, óxido de ferro e óxido de alumínio não deve ser inferior a 70\% (APRIANTI, 2017). A análise da GA demonstra que 95,9\% de sua composição está distribuída entre os principais óxidos descritos pela ASTM C618 (ASTM, 2019).

Segundo Silva (2018), a sílica, quando presente no estado amorfo, está diretamente relacionada à quantidade de hidróxido de cálcio $(\mathrm{CH})$ que pode ser consumido pela atividade pozolânica; sendo assim, é possível que esse rejeito atue como pozolana ao ser aplicado em matrizes cimentícias. Outro fator observado foi a expressiva quantidade de $\mathrm{Al}_{2} \mathrm{O}_{3}, \mathrm{que}$ pode reagir com a cal no momento da hidratação, resultando na formação de aluminatos de cálcio e reduzindo, assim, a quantidade de Portlandita $\left[\mathrm{Ca}(\mathrm{OH})_{2}\right]$ na matriz. Este efeito pode ser favorável ao melhor desempenho de um concreto em meios agressivos, como incêndios e ataques químicos (OLLIVIER e VICHOT, 2014).

Tabela 3 - Teores dos óxidos por FRX

\begin{tabular}{c|c|c|c|c|c}
\hline $\begin{array}{c}\mathrm{SiO}_{2} \\
(\%)\end{array}$ & $\begin{array}{c}\mathrm{Al}_{2} \mathrm{O}_{3} \\
(\%)\end{array}$ & $\begin{array}{c}\mathrm{Fe}_{2} \mathrm{O}_{3} \\
(\%)\end{array}$ & $\begin{array}{c}\mathrm{TiO}_{2} \\
(\%)\end{array}$ & $\begin{array}{c}\mathrm{K}_{2} \mathrm{O} \\
(\%)\end{array}$ & $\begin{array}{c}\text { Outros* } \\
(\%)\end{array}$ \\
\hline 58,6 & 33,1 & 4,2 & 1,8 & 1,0 & 1,3 \\
\hline
\end{tabular}

* Elementos com teores abaixo de $1 \%$

O difratograma da amostra de GA segue apresentado na Figura 3. As fases minerais encontradas nesse material foram quartzo e caulinita, sendo também observado um percentual de griceita, uma vez que esse composto foi adicionado à amostra para estimativa do presente teor de amorfos.

De acordo com o refinamento quantitativo realizado pelo método de Rietveld, a composição mineralógica da GA consiste em $26,0 \%$ de quartzo $\left(\mathrm{SiO}_{2}\right), 38,3 \%$ de caulinita $\left[\mathrm{Al}_{2} \mathrm{Si}_{2} \mathrm{O}_{5}(\mathrm{OH})_{4}\right]$ e $35,7 \%$ de material amorfo ou desconhecido. Uma vez determinado pela fluorescência de Raios-X um teor de 58,6\% de sílica, pode-se inferir que parte da matéria não-cristalina é constituída por sílica amorfa. A presença de fases amorfas é favorável à finalidade de material cimentício suplementar, pois estruturas com essa característica são mais reativas do que as estruturas cristalinas (SILVA, SILVA, et al., 2018) (MASSAZZA, 1993). 


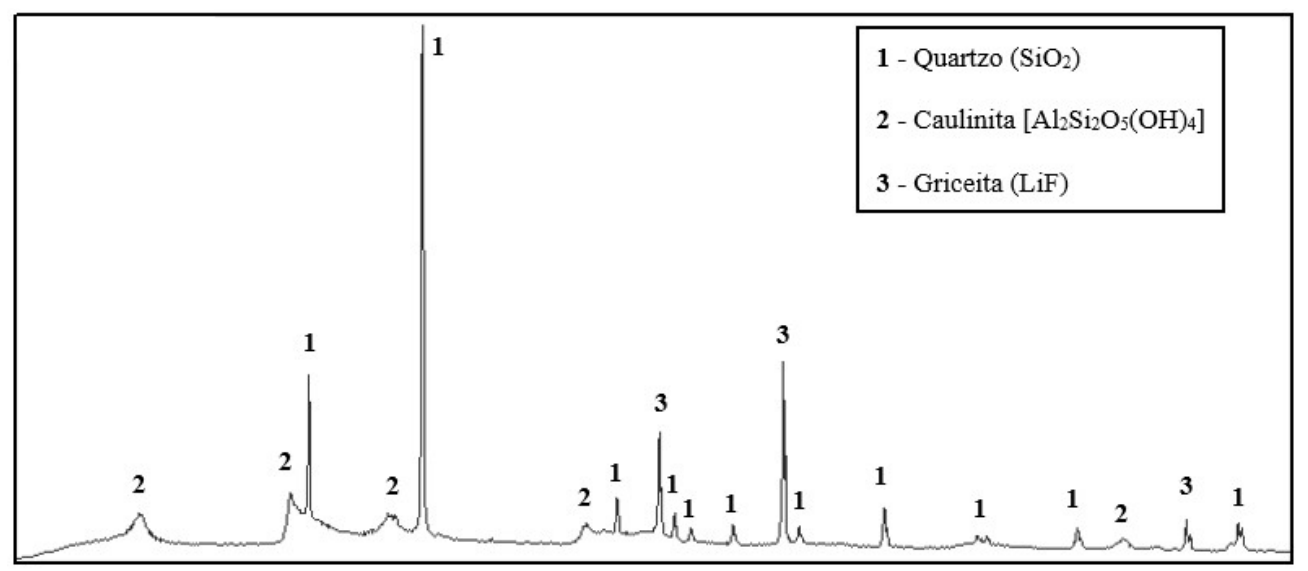

Figura 3 - DRX da goma de areia

Os resultados da análise termogravimétrica e diferencial (TG/DTA) são apresentados na Figura 4, a seguir. Verifica-se que a decomposição térmica da GA apresentou duas faixas de perda significativa de massa, indicadas nos intervalos de temperatura entre $0-130^{\circ} \mathrm{C}$ e $400-500^{\circ} \mathrm{C}$. A perda de massa de $1,9 \%$, acompanhada de um pico endotérmico referente ao intervalo de temperatura até $130^{\circ} \mathrm{C}$, está relacionada à perda de água livre e adsorvida nas partículas (OLLIVIER e VICHOT, 2014).

O segundo intervalo, com perda de massa de 6,2\% e um expressivo pico endotérmico próximo de $450^{\circ} \mathrm{C}$, representa a desidroxilação da caulinita presente neste resíduo (BALEK e MURAT, 1996). Próximo aos $925^{\circ} \mathrm{C}$, sem perda significativa de massa, ocorre um pico exotérmico, atribuído à formação de 'precursores' ( $\gamma$-alumina ou espinélio de Alumínio-Silício e silicatos amorfos) das fases de altas temperaturas (mulita e cristobalita) (BAKOLAS, AGGELAKOPOULOU, et al., 2006) (MAYORAL, IZQUIERDO, et al., 2001) (BERNAL, JUENGER, et al., 2017).

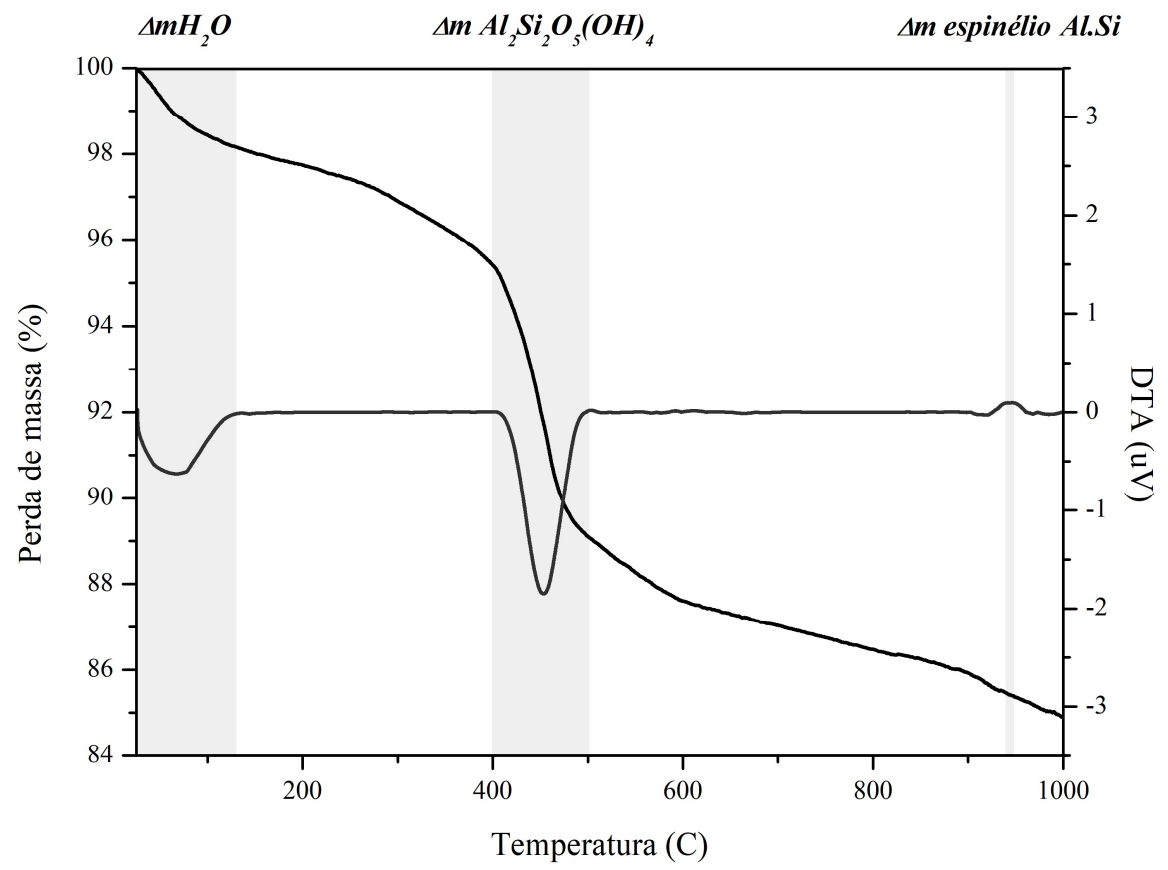

Figura 4 - Análise Térmica Simultânea TG/DTA da goma de areia

A análise TG e DTA corrobora os resultados apresentados pela difração de Raios-X, de maneira a indicar que o resíduo analisado pelo presente trabalho possui em sua composição uma predominância do mineral caulinita. 


\subsection{Caracterização dos concretos}

A caracterização física dos concretos no estado endurecido (Figura 5) demonstrou um aumento de apenas 2,9\% na massa específica dos corpos de prova com adição de goma de areia, indicando que não haveria prejuízo quanto ao peso próprio no caso de emprego em estuturas convencionais. O índice de vazios e a absorção de água sofreram uma redução de 19,6\% e $23,4 \%$, respectivamente; o que aponta um refinamento na estrutura de poros, que consiste em uma maior desconexão entre os vazios da matriz e implica na redução da percolação de agentes agressivos no concreto produzido com goma de areia.

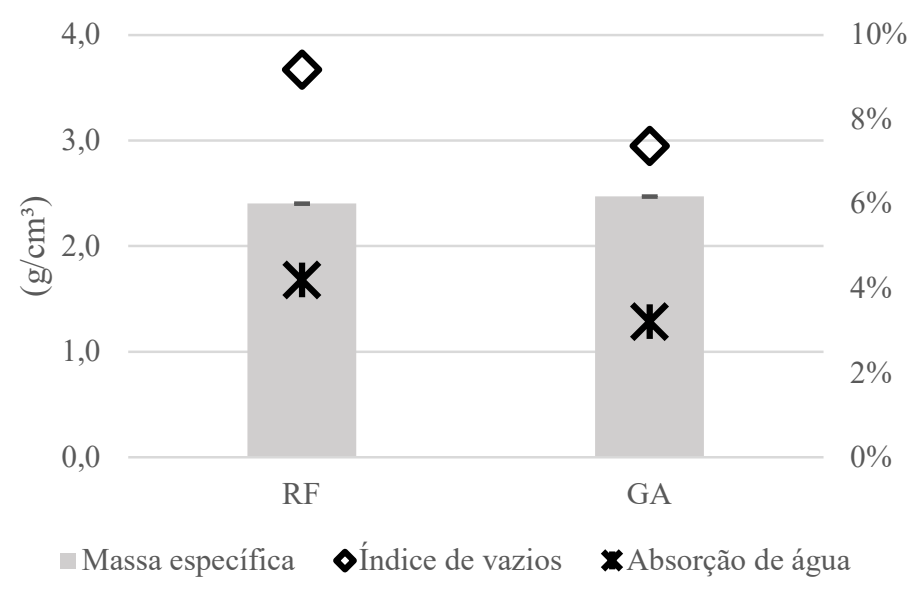

Figura 5 - Propriedades físicas dos concretos no estado endurecido

Os resultados da caracterização mecânica dos concretos (Figura 6) apresentaram um incremento de 19,2\% na resistência à tração e 18,5\% na resistência à compressão, o que sugere interação da goma de areia com o cimento; seja através da formação de novos compostos resistentes ou da nucleação, reforçando o proposto pelas análises física, química e mineralógica do material. O resultado de VPU aponta um aumento de 4,6\% para os concretos com GA, corroborando, também, todas as análises procedidas no estado endurecido ao indicar uma matriz mais coesa e com menores descontinuidades.

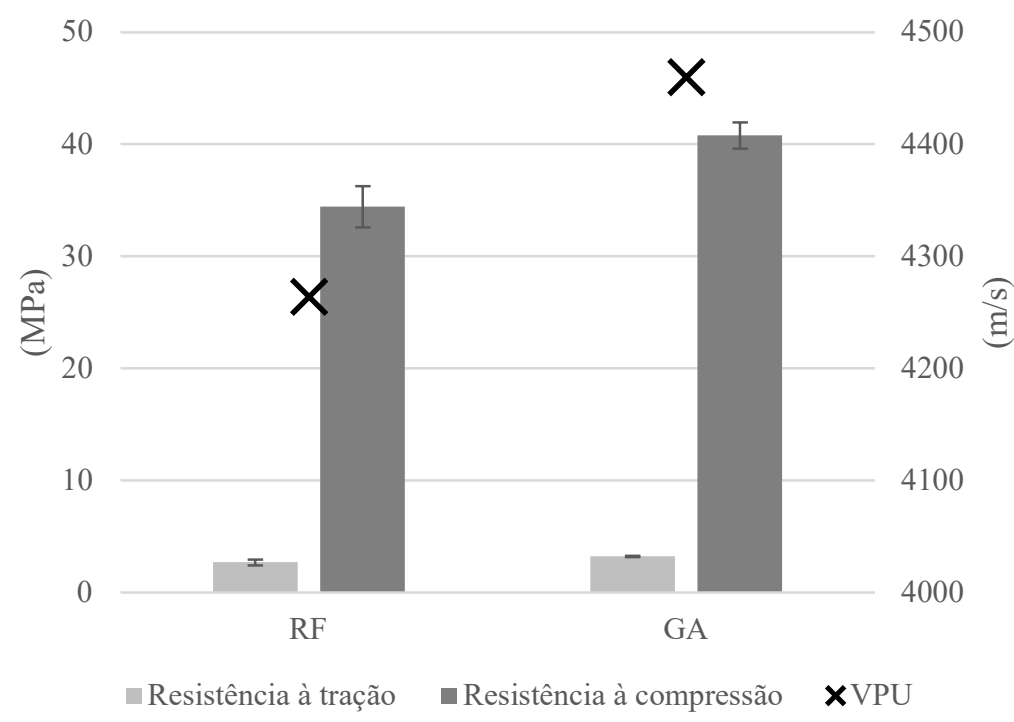

Figura 6 - Propriedades mecânicas e VPU dos concretos no estado endurecido

Partindo destes resultados, é válido afirmar que a aplicação da goma de areia em concretos não só é tecnicamente viável, como permite a obtenção de uma matriz mais resistente tanto mecânica quanto fisicamente, com características associadas à maior durabilidade e menor suscetibilidade a patologias decorrentes de cargas estruturais ou de agentes externos. 


\section{CONCLUSÃO}

De acordo com os resultados obtidos, a incorporação da goma de areia em matrizes cimentícias se mostrou bastante viável; tanto em termos de suas propriedades físicas, quanto de suas propriedades químicas e mineralógicas. A GA possui $90 \%$ das partículas abaixo de $20 \mu \mathrm{m}$ sem a necessidade de cominuição em moinho. Desta maneira, de acordo com as normas referentes, este material poderia ser incorporado a matrizes cimentícias de forma sustentável, sem consumo adicional de energia neste processo. A alta concentração de $\mathrm{Al}_{2} \mathrm{O}_{3}$, na forma de caulinita, presente neste rejeito é semelhante a alguns SCMs já difundidos industrialmente, como o metacaulim. Adicionalmente, um alto teor de $\mathrm{Al}_{2} \mathrm{O}_{3}$ pode ser favorável à formação de compostos que melhorem a resistência de concretos em meios agressivos. Portanto, sugere-se que a GA possa ser utilizada como adição mineral em matrizes cimentícias de modo geral, com o objetivo de produzir elementos construtivos mais duráveis com propriedades mais adequadas caso submetidos a condições adversas.

\section{AGRADECIMENTOS}

O presente trabalho foi realizado com apoio da Coordenação de Aperfeiçoamento de Pessoal de Nível Superior - Brasil (CAPES) - Código de Financiamento 001. Os autores também agradecem à FAPEMIG, CNPq e UFOP pelo apoio para a realização e apresentação dessa pesquisa. Somos gratos ainda pela infraestrutura e colaboração do Grupo de Pesquisa em Resíduos Sólidos - RECICLOS - CNPq.

\section{REFERÊNCIAS}

ABNT. NBR NM 67 - Concreto - Determinação da consistência pelo abatimento do tronco de cone. Rio de Janeiro. 1998.

ABNT. NBR 9778: Argamassa e concreto endurecidos - Absorção de água, índice de vazios e massa específica. Associação Brasileira de Normas Técnicas. Rio de Janeiro. 2005.

ABNT. NBR NM 45: Agregados - Determinação da massa unitária e do volume de vazios. Rio de Janeiro. 2006.

ABNT. NBR 15894. Metacaulim para uso com cimento Portland em concreto, argamassa e pasta: requisitos. Rio de Janeiro. 2010.

ABNT. NBR 7222: Concreto e argamassa - Determinação da resistência à tração por compressão diametral. Associação Brasileira de Normas Técnicas. Rio de Janeiro. 2011.

ABNT. NBR 5752. Materiais pozolânicos: determinação do índice de desempenho com cimento Portland aos 28 dias. Rio de Janeiro. 2014.

ABNT. NBR 5738: Concreto - Procedimento para moldagem e cura de corpos de prova. Associação Brasileira de Normas Técnicas. Rio de Janeiro. 2015.

ABNT. NBR 16605:2017. Cimento Portland e outros materiais em pó - Determinação da massa específica. Rio de Janeiro. 2017.

ABNT. NBR 5739: Concreto - Ensaio de compressão de corpos de prova cilíndricos. Associação Brasileira de Normas Técnicas. Rio de Janeiro. 2018.

ABNT. NBR 8802: Concreto endurecido - Determinação da velocidade de propagação de onda ultrassônica. Associação Brasileira de Normas Técnicas. Rio de Janieiro. 2019.

ANDRADE, H. D. Carbonatação em concretos de escória de aciaria. Dissertação de mestrado. Universidade Federal de Ouro Preto: Programa de Pós Graduação em Engenharia Civil (PROPEC). 2018.

ANEPAC. Associação Nacional das Entidades de Produtores de Agregados para Construção, 2019. Disponivel em: $<$ https://www.anepac.org.br/>. Acesso em: abril 2019. 
APRIANTI, E. A huge number of artificial waste material can be supplementary cementitious material (SCM) for concrete production e a review part II. Journal of Cleaner Production, p. 4178-4194, 2017.

ARVANITI, E. C. et al. Determination of particle size, surface area, and shape of supplementary cementitious materials by different techniques. Materials and Structures, v. 48, p. 3687-3701, 2015.

ASTM. C618-19: Standard Specification for Coal Fly Ash and Raw or Calcined Natural Pozzolan for Use in Concrete. [S.1.]. 2019.

BAKOLAS, A. et al. Evaluation of pozzolanic activity and physicomechanical characteristics in metakaolin-lime pastes. Journal of Thermal Analysis and Calorimetry, v. 84, p. 157-163, 2006.

BALEK, V.; MURAT, M. The emanation thermal analysis of kaolinite clay minerals. Thermochimica Acta, v. 282/283, p. $385-397,1996$.

BERNAL, S. A. et al. Characterization of supplementary cementitious materials by thermal analysis. Materials and Structures, v. 50, n. 26, p. 1-13, 2017.

BITAR, O. Y.; IYOMASA, W. S.; JR., M. C. Geotecnologia: tendências e desafios. São Paulo em Perspectiva, v. 14, n. 1, p. 78-90, 2000.

CARVALHO, J. M. F. D. et al. Low Environmental Impact Cement Produced Entirely from Industrial and Mining Waste. J. Mater. Civ. Eng, v. 31, 2019.

CHAVES, A. P.; WHITAKER, W. Operações de beneficiamento de areia. In: construção civil. Rio de Janeiro: CTEM/MCTI, 2012. p. 197-219.

Manual de agregados para a

COSTA, L. C. B. Desempenho de concretos de escória de aciaria frente a ataque de cloretos. Dissertação de mestrado. Universidade Federal de Ouro Preto: Programa de Pós Graduação em Engenharia Civil (PROPEC). 2019.

DNPM. Sumário Mineral. Departamento Nacional de Produção Mineral, Brasília, 2016.

FERREIRA, G. C. Avaliaçao dos mercados produtor e consumidor de areia industrial no Estado de Sao Paulo. Geociencias, v. 16, p. 433-466, 1997.

FERREIRA, G. C.; DAITX, E. C. A mineração de areia industrial na Região Sul do Brasil. Revista Escola de Minas, v. 56, n. 1, p. 59-65, 2003.

FIGUEIREDO, B. R.; CAPITANI, E. M. D.; GITAHY, L. C. Exposição humana à contaminação por chumbo e arsênio no Vale do Ribeira (SP-PR). Encontro da ANPPAS, Indaiatuba, 02 maio 2004. 1-13.

JUENGER, M. C. G.; SIDDIQUE, R. Recent advances in understanding the role of supplementary cementitious materials in concrete. Cement and Concrete Research, p. 71-80, 2015.

JUENGER, M. C. G.; SNELlingS, R.; BERNAL, S. A. Supplementary cementitious materials: New sources, characterization, and performance insights. Cement and Concrete Research, p. 257-273, 2019.

KORPA, A.; KOWALD, T.; TRETTIN, R. Hydration behaviour, structure and morphology of hydration phases in advanced cement-based systems containing micro and nanoscale pozzolanic additives. Cement and Concrete Research, v. 38, p. 955-962, 2008.

LEIBNIZ ASSOCIATION. Inorganic Crystal Structure Database, 2019. Disponivel em: <https://www.fizkarlsruhe.de/en/produkte-und-dienstleistungen/inorganic-crystal-structure-database-icsd>.

LOTHENBACH, B.; SCRIVENER, K.; HOOTON, R. D. Supplementary cementitious materials. Cement and Concrete Research, p. 1244-1256, 2011. 
LOTTERMOSER, B. G. Mine Wastes: Characterization, Treatment and Environmental Impacts. Journal of Cleaner Production, v. 14, p. 1176-1177, 2006.

LUZ, A. B. D.; LINS, F. F. Areia industrial. In: Rochas e minerais industriais: usos e especificações. Rio de Janeiro: CTEM, 2005. p. 107-126.

MASSAZZA, F. Pozzolanic cements. Cement \& Concrete Composites, v. 15, p. 185-214, 1993.

MAYORAL, M. C. et al. Aluminosilicates transformations in combustion followed by DSC. Thermochimica Acta, v. 373 , p. 173-180, 2001.

MECHI, A.; SANCHES, D. L. Impactos ambientais da mineração no Estado de São Paulo. Estudos Avançados, v. 24, n. 68 , p. 209-220, 2010.

OLLIVIER, J.-P.; VICHOT, A. Durabilidade do concreto: bases científicas para a formulação de concretos duráveis de acordo com o ambiente. 1. ed. [S.1.]: Ibracon, 2014.

RAHLA, K. M.; MATEUS, R.; BRAGANÇA, L. Comparative sustainability assessment of binary blended concretes using Supplementary Cementitious Materials (SCMs) and Ordinary Portland Cement (OPC). Journal of Cleaner Production, p. 445-459, 2019.

SANTOS, P. D. S. Tecnologia de argilas: aplicações. São Paulo: Blucher, 1975.

SILVA, K. D. C. et al. Rock Wool Waste as Supplementary Cementitious Material for Portland Cement-Based Composites. ACI Materials Journal, v. 15, n. 5, p. 653-661, 2018.

SNELLINGS, R. Assessing, Understanding and Unlocking Supplementary Cementitious Materials. RILEM Technical Letters, p. 50-55, 2016.

SNELlingS, R.; MERTENS, G.; ELSEN, J. Supplementary Cementitious Materials. Reviews in Mineralogy \& Geochemistry, v. 74, p. 211-278, 2012.

WHITAKER, W.; BRAGA, J. M. S.; CHAVES, A. P. Prospeccion de un tramo de rio para el planeo de la mineracion de arenas. Saltas. 2002.

YANG, K.-H. et al. Effect of supplementary cementitious materials on reduction of CO2 emissions from concrete. Journal of Cleaner Production, p. 774-783, 2015.

YOUNG, G.; YANG, M. Preparation and characterization of Portland cement clinker from iron ore tailings. Construction and Building Materials, v. 197, p. 152-156, 2019. 